\title{
Lack of knowledge in health professionals: a barrier to providing information to patients?
}

\author{
D K Smith, J Slack, R W Shaw, T M Marteau
}

\begin{abstract}
Objective-To assess obstetricians' and midwives' knowledge of routine prenatal screening tests for fetal abnormality and factors associated with such knowledge. Design-Questionnaire assessment of antenatal clinic staff.

Setting-Six hospitals within the United Kingdom (four district general hospitals in London, one district general hospital in Wales, and one teaching hospital in Wales), offering routine prenatal screening tests.

Subjects-29 obstetricians and 97 midwives were invited to participate, of whom 21 and 70 respectively responded to the questionnaire.

Main measures-Knowledge of prenatal tests, according to 19 item multiple choice questionnaire, reluctance to disclose uncertainty, and clinical experience.

Results-The overall response rate was $72 \%(91 / 126)$. In all, $43 \%$ of midwives and $14 \%$ of obstetricians obtained correct responses on fewer than half the items. Reluctance to disclose uncertainty to patients was associated in obstetricians with having less knowledge about prenatal testing $(\mathbf{r}=-0.50 ; \quad p<0.025$, Pearson product moment correlation) and in midwives with more clinical experience $(\mathrm{r}=0.43 ; \mathrm{p}<0.001)$.

Conclusions-Lack of knowledge and greater clinical experience seem to be important barriers to providing patients with information about prenatal screening tests.

(Quality in Health Care 1994;3:75-78)
\end{abstract}

Genetics Researc

Group, United

Medical and Dental

Schools (UMDS),

Guy's Campus,

London SE1 9RT

D K Smith, research

psychologist

T M Marteau, director

Department of

Clinical Genetics,

Royal Free Hospital

School of Medicine,

London NW3

J Slack, senior lecturer

Department of

Obstetrics and

Gynaecology,

University of Wales

College of Medicine,

Cardiff CF4

$\mathrm{R}$ W Shaw, professor

Correspondence to:

Dr Marteau

Accepted for publication

23 June 1994

\section{Introduction}

The most frequent complaint among patients is that professional staff providing their care fail to give sufficient information about their condition or treatment. ${ }^{1}$ We know little, however, about the reasons for this.

There is some evidence to suggest that health professionals may lack sufficient knowledge to provide enough information. ${ }^{2}$ Another possible explanation for giving poor information is an unwillingness to disclose uncertainties about health care or test results. This is particularly pertinent when presenting screening tests that provide probabilistic rather than categorical results. ${ }^{34}$ There is, however, widespread agreement that patients need to be informed about the uncertainties of screening, such as the possibility of false negative or false positive results. ${ }^{45}$ One sample of a clinical situation in which patients may receive inadequate information is the presentation of routine prenatal screening tests for fetal abnormality. ${ }^{67}$ Health professionals often neglect to give women the information necessary for them to make informed decisions about whether to undergo tests. When information is given, it is sometimes inaccurate. ${ }^{67}$

This study investigates the relation between knowledge of prenatal tests, attitudes towards communicating information about them, and clinical experience among obstetricians and midwives.

\section{Subjects and methods}

SAMPLE

We invited all obstetricians and midwives from six hospitals in the United Kingdom to take part in this study. The participating hospitals had all agreed to take part in a larger intervention study (D K Smith et al, unpublished): four were district general hospitals within greater London, one was a district general hospital in Wales, and one was a teaching hospital in Wales. Two hospitals routinely offered maternal serum $\alpha$ fetoprotein (AFP) testing to pregnant women, one to screen for risk of spina bifida alone and the other to screen for risks of both spina bifida and Down syndrome. Two hospitals offered the double test (AFP and human chorionic gonadotrophin, or hCG) and two offered the triple test (AFP, hCG and unconjugated oestriol) to screen for risks of both spina bifida and Down syndrome.

A total of 29 obstetricians and 97 midwives were invited to participate, ranging in seniority from newly qualified nurses to consultants. The number of years since qualifying ranged from less than one year to 38 years (mean 11.9 years).

\section{PROCEDURE}

Questionnaires were handed personally to health professionals in their clinics. Those subjects who did not return their questionnaires were sent further copies, followed by a telephone reminder if the questionnaires had still not been received after two weeks.

\section{MEASURES}

Knowledge was assessed with a 19 item multiple choice questionnaire assessing knowledge about routine prenatal tests for fetal abnormality. The items were selected to cover the information widely considered to be important for women to have in making informed decisions about routine prenatal 
screening tests. ${ }^{589}$ The questionnaires differed slightly between hospitals according to the type of screening test offered. Two points were awarded for each correct answer, giving a maximum total of 38 points. One point was awarded for answers that were considered close enough to the correct answer to be clinically appropriate. Table 1 shows an example of the knowledge questionnaire and its scoring key. Factor analysis of the questionnaire showed it to be unidimensional, with a Cronbach's $\alpha$ coefficient of 0.59 .

Reluctance to disclose uncertainty was measured with the scale developed by Gerrity et al, ${ }^{9}$ which comprises nine items, each scored on a scale ranging from 1 (strongly agree) to 6 (strongly disagree). The score range therefore is 9 to 54 , with high scores denoting a greater reluctance to disclose uncertainty to patients. The norm, based on responses of 428 North American physicians, is: 23 (SD 6, range 9-39).
Clinical experience was assessed by asking subjects how many years they had been qualified in obstetrics or midwifery.

\section{Results}

Of the 126 obstetricians and midwives invited to participate, 21 obstetricians and 70 midwives from all six hospitals completed the study, a response rate of $72 \%$.

Total scores for knowledge ranged from 5 to $32($ mean $(\mathrm{SD})$ score $=20 \cdot 1(5 \cdot 1))$. The mean score of obstetricians was significantly higher than that of midwives $(24 \cdot 7(4 \cdot 6) \approx 19 \cdot 5(4 \cdot 6)$, $t=-4.5, \mathrm{df}=89 ; \mathrm{p}<0.001)$. Thirty midwives $(43 \%)$ and three obstetricians $(14 \%)$ obtained correct responses on fewer than half the items. The proportion of obstetricians and midwives selecting each possible response to all questions is shown in table 1 . For some items only a small proportion of health professionals selected the correct response - for example, less than a quarter of obstetricians and

Table 1 Example of knowledge questionnaire, with scoring key and proportion of obstetricians and midwives selecting each response

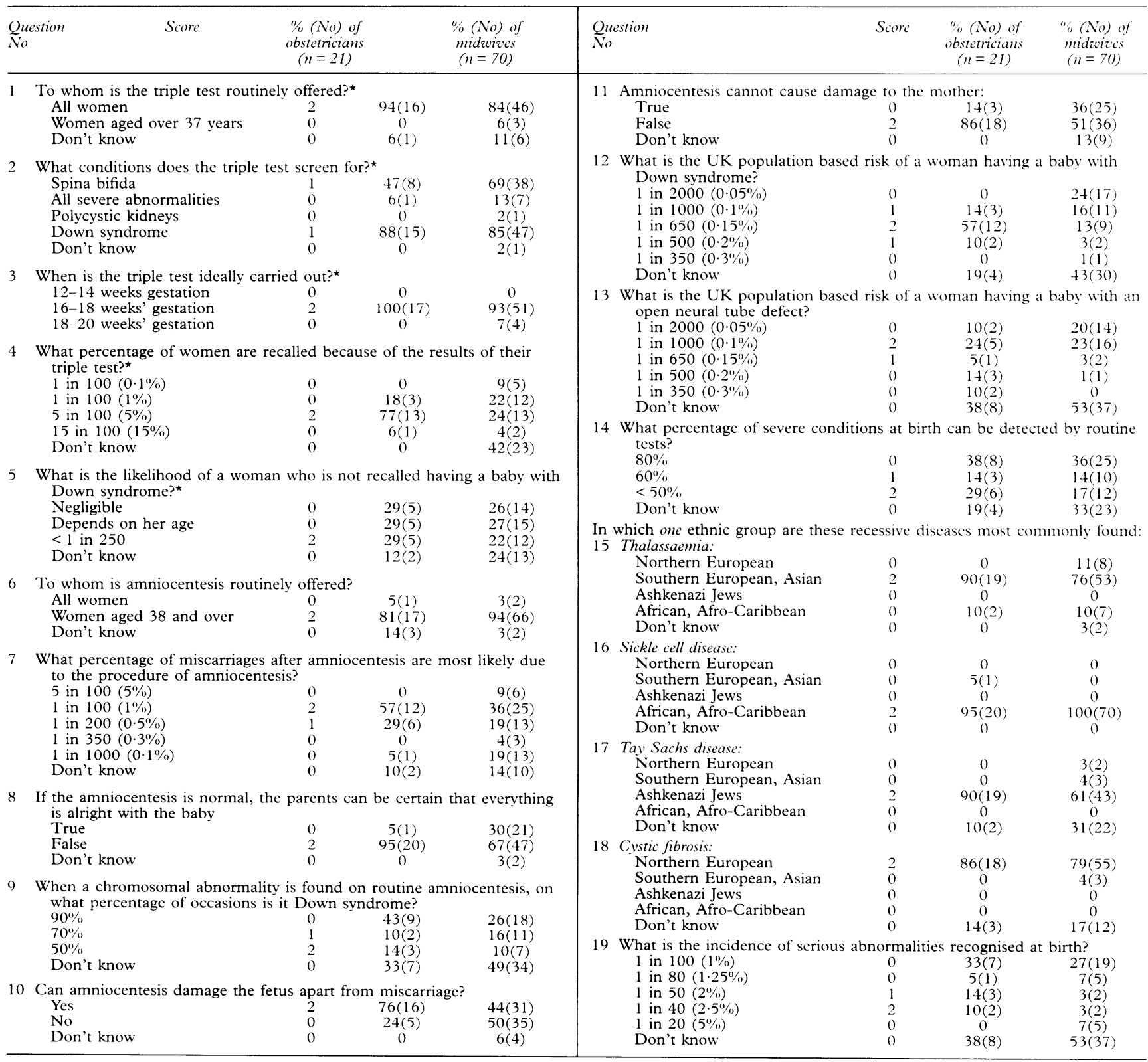

${ }^{\star}$ For questions $1-5$ total number of obstetricians $=17$, total number of midwives $=55$. 
Table 2 Pearson product moment correlations between knowledge about prenatal tests, reluctance to disclose uncertainty, and number of years since qualifying for 21 obstetricians and 70 midwives

\begin{tabular}{lccccc}
\hline & \multicolumn{2}{c}{ Reluctance to disclose uncertainty } & & \multicolumn{2}{c}{ No of years since qualifying } \\
\cline { 2 - 3 } & Obstetricians & Midwives & & Obstetricians & Midwives \\
\hline Knowledge score & $-0.50^{\star}$ & -0.06 & & -0.07 & 0.09 \\
Reluctance to disclose uncertainty & & & & 0.25 & $0.43^{\star \star}$ \\
\hline${ }^{\star} \mathrm{p}<0.025$. & & & & \\
${ }^{\star} \mathrm{p}<0.001$. & & & & &
\end{tabular}

midwives combined knew the likelihood of a woman who is not recalled on her screening test result having a baby with Down syndrome (question 5).

Table 2 shows Pearson product moment correlations between scores for knowledge and for reluctance to disclose uncertainty and number of years since qualifying for both midwives and obstetricians. For obstetricians, being less knowledgeable was associated with a greater reluctance to disclose uncertainty $(\mathrm{r}=-0.50 ; \quad \mathrm{p}<0.025)$. Knowledge was unrelated to years since qualifying in either obstetrics or midwifery. Midwives with greater experience were more reluctant to disclose uncertainty $(r=0.43 ; p<0.001)$.

\section{Discussion}

Based on responses to a knowledge questionnaire, we infer that knowledge of routine prenatal screening tests is poor in $43 \%$ of midwives and $14 \%$ of obstetricians for whom presenting prenatal screening tests is part of their routine clinical work. Among obstetricians, reluctance to disclose uncertainty to patients is negatively associated with knowledge about prenatal testing. Among midwives, greater reluctance is associated with more clinical experience.

The lack of knowledge among midwives is similar to that recorded nearly 10 years ago in a Swedish study. ${ }^{2}$ One reason for suboptimal knowledge of a relatively new test is that there is no formal procedure for educating staff about new clinical developments. Recognising this problem, the Royal College of Obstetricians and Gynaecologists has recommended that one member of staff be designated such a role in each clinic. ${ }^{8}$ It is not known how many clinics are following this recommendation.

However, some caution is needed in interpreting the results of responses to questionnaires on knowledge. The validity of responses depends how the scale is constructed. Multiple choice questionnaires are often problematic in that apparent knowledge may be manipulated by the wording of questions asked and by the range of possible responses given. This may partly explain the higher scores obtained by obstetricians, who may be more familiar than midwives with multiple choice questionnaires. Similarly, caution is needed in interpreting responses to questionnaires concerning attitudes towards giving information. The extent to which such attitudes predict behaviour has yet to be established.

Even if a questionnaire can validly measure knowledge, the domain of knowledge sampled needs to be clinically relevant. The questionnaire used in this study assessed knowledge about aspects of prenatal tests that are considered necessary to inform women's decision making, as well as to prepare them for possible adverse outcomes of tests. ${ }^{8}$ Women should know, for example, their chance of miscarrying after amniocentesis if they are to make an informed choice about whether or not to undergo the procedure. Similarly, they need to know the chance of being recalled with a positive result on a screening test so that they can be prepared for this outcome.

Even if knowledge is perfect, other factors are important in predicting what information is communicated - for example, beliefs about the importance of information to decision making and attitudes towards giving information to patients. Those with high scores on the reluctance to disclose uncertainty scale are less likely to discuss the meaning of screening test results with patients, as these provide information that is uncertain. The lower an obstetrician's level of knowledge, the more reluctant he or she is to disclose uncertainty. This reluctance may reflect an appropriate lack of confidence in their knowledge among those with less knowledge about prenatal tests. If so, then one solution to increasing the amount of information health professionals give is to ensure that their own knowledge is sufficient. For midwives, however, knowledge did not predict willingness to disclose uncertainty whereas number of years since qualifying predicted reluctance to disclose uncertainty. This association has several possible explanations. Firstly, it may reflect willingness to admit to being reluctant to give patients information. Increasingly, guidelines for clinical practice encourage the provision of information to patients. Talking about the possible negative effects of such guidelines may be done only by more senior health professionals (for an example, see reference 10). Another explanation is that these differences may stem from different philosophies prevailing during training for more experienced, as opposed to less experienced, midwives. Alternatively, reluctance may have been reinforced by positive clinical experiences of not admitting uncertainty, or negative clinical experiences of admitting uncertainty. A further possible explanation is that years since qualifying may not be the same as clinical experience. Some midwives may have had career breaks or worked part time. This information was not collected during the study.

The need for health professionals to develop good communication skills is now widely recognised. ${ }^{11}$ The results of our study highlight some of the factors that need to be considered in attempting to improve these skills, in particular to increase the information that health professionals routinely provide to patients. Although the educational needs of staff may be identified relatively easily, understanding the attitudes that underlie some of their practices may be a prerequisite to achieving change, and these attitudes require more study.

This study was funded by the Medical Research Council. We gratefully acknowledge communications wtih Chris McManus in developing this paper. 
1 Lev P. Communicating zith patients. London: Croom Helm, 1988.

2 Sanden M-L. Midwives' knowledge of the alphafetoprotein test. Fournal of Psychosomatic Obstetrics and Gynaecology

3 Garrett TM, Baillee HW, Garrett RM. Health care ethics: principles and problems. New Jersey: Prentice Hall, 1993.

4 Royal College of Physicians. Prenatal diagnosis and genetic screening: community and service implications. London: Royal College of Physicians of London, 1989.

5 Nuffield Council on Bioethics. Genetic screening: ethical issues. London: Nuffield Council on Bioethics, 1993.

6 Marteau T, Slack J, Kidd J, Shaw R. Presenting a routine screening test in antenatal care: practice observed. Public Health 1992;106:131-41.
7 Marteau TM, Plenicar M, Kidd J. Obstetricians presenting amniocentesis to pregnant women: practice observed. amniocentesis to pregnant women: practice observed.
foumal of Reproductice and Infant Psichology 1993;11:3-10.

8 Royal College of Obstetricians and Gynaccologists. Report of the RCOG working party on biochemical markers and the detection of Dozen's sindrome. London: RCOG Press,

9 Gerrity MS, Devellis RF, Earp JA. Physicians" reactions to uncertainty in patient care. A new measure and new insights. Med Care 1990;28:724-36.

() Souhami RL, Tobias JS. Fully informed consent can be needlessly cruel. BMF 1993;307:1199-201.

11 Simpson .M, Buckman R, Stewart M, Maguire P, L ipkin M, Novac D, et al. Doctor-patient communication: the Toronto consensus statement. BM7 1991;303:1385 7 . 